\title{
Use of the Femtosecond Lasers in Ophthalmology
}

\author{
Anna M Roszkowska ${ }^{1,2, *}$, Mario Urso ${ }^{1}$, Alberto Signorino ${ }^{1}$, Pasquale Aragona ${ }^{1}$ \\ ${ }^{1}$ Ophthalmology Section, Department of Surgery, University Hospital of Messina, 98124 Messina, Italy. \\ ${ }^{2}$ Department of Ophthalmology, Andrzej Frycz Modrzewski Kraków University, Cracow, Poland.
}

\begin{abstract}
Femtosecond laser (FSL) is an infrared laser with a wavelength of $1053 \mathrm{~nm}$. FS laser works producing photodisruption or photoionization of the optically transparent tissue such as cornea. Currently FS lasers have a wide range of applications in ophthalmic surgery. They are used above all in corneal surgery in refractive procedures and keratoplasty, and recently in cataract surgery. The use of the FSL in corneal refractive surgery includes LASIK flap creation, astigmatic keratotomy, Femtosecond Lenticule Extraction (FLEx), Small Incision Lenticule Extraction (SMILE) and channels creation for implantation of the intrastromal corneal rings. As to the corneal grafting, the FS lasers are used in laser-assisted anterior and posterior lamellar keratoplasty and customized trephination in the penetrating keratoplasty. FS Laser Assisted Cataract Surgery (FLACS) includes capsulorrhexis and nuclear fragmentation that enhance safety and efficacy of the procedure.
\end{abstract}

\section{Introduction}

Since the ocular damage subsequent to exposure to the intense sunlight during eclipse was noticed, the idea for the development of therapeutic lasers opened the new era in ophthalmology. Actually, different lasers are used in diagnostic and therapeutic ophthalmic procedures. It is due to their particular characteristics that distinguish them from conventional light sources such as their directivity, pulsed mode and monochromaticity, with different wavelength absorbed by different ocular structures that permits to obtain their final effects on tissues.

First lasers were applied to treat diabetic retinopathy. They worked in blue/green part of light spectrum (488$515 \mathrm{~nm}$ ) being absorbed by posterior ocular layers such as pigmented retinal epithelium, retinal vessels and blood pigment. Successively other devices were introduced and nowadays numerous lasers work with different wavelengths reaching almost all ocular structures and treating various ocular disorders. The use of lasers in ophthalmology is based on their three fundamental tissue effects: thermal, photoablative and mechanical. Thermal effects, obtained with lasers working with 400-700 $\mathrm{nm}$ wavelength, are exploited to perform tissue photocoagulation in several retinal diseases, whereas photoablation, obtained with excimer lasers working with $193 \mathrm{~nm}$ wavelength, permits to modify the anterior corneal shape by evaporation of the corneal stroma and so is used successfully in corneal refractive surgery.

Mechanical effects are obtained by two type of lasers working with different modalities such as Nd:YAG and

Corresponding author: aroszkowska@unime.it femtosecond laser. Nd:YAG emits light with a wavelength in the infrared of $1064 \mathrm{~nm}$, and produces mechanical effect by plasma creation. It is mostly used to treat the secondary cataract practicing a posterior capsulotomy. However it is also used to perform peripheral iridotomy in acute angle closure glaucoma.

The femtosecond lasers work in deep infrared absorbed by superficial ocular layers that permits to use them in corneal and anterior segment surgery.

\section{Femtosecond lasers.}

The most recent lasers approved for ophthalmic procedures are the Femtosecond lasers (FSL), working in the deep infrared and characterized by ultra-short pulses (1028-1053 nm) in the domain of femtoseconds $\left(1 \mathrm{fs}=10^{-15} \mathrm{~s}\right)$. The final effect is a perfect cut of tissues obtained by cavitation. FS belongs to the category of ultrafast lasers or ultra short pulse lasers with beam diameter less than 8 micron. Characteristic of the absorption of FS wavelength permits to use them in corneal and anterior segment surgery. Their capacity to perform multiple geometric cuts, with horizontal, vertical or oblique pattern, permitted to use them in refractive, corneal and cataract surgery. Thousands of laser spots are applied uninterruptedly to form a resection plane, creating multiple cavitational bubbles and thus generating the surgical effects (Fig. 1). Through a photodisruption process that rapidly executes thousands of impulses close to each other, it creates a perfect cut. Ultra-short pulse duration delivers laser energy with preserving the surrounding tissues. Lasers energy is absorbed by targeted tissues with rapidly expanding plasma formation with creation of cavitation 
bubbles separating the tissue plane. The cavity bubble results in a single spot with the size of about 5 microns and is constituted of carbon dioxide and water vapor. This bubble expands several times and the final dimensions depend on the applied energy (Fig. 2). The application of this technology in the field of ocular microsurgery has found various uses over time. Since the year 2000 the FSL have been used in lamellar and perforating corneal transplantation and in 2001 the FDA (USA Food and Drug Administration) approved it for the creation of the corneal flap in refractive surgery. Today it is also used to correct the corneal astigmatism by curve keratotomy, and in the management of keratoconus with intrastromal rings. The first use of the Femtolaser in cataract surgery had place in 2008 in Budapest and in 2009 the FDA approved its employment for this procedure. The platforms actually available for Femtolaser Assisted Cataract Surgery are: LenSx (Alcon, Fort Worth, Texas, USA); LensAR (Orlando, Florida, USA); Catalys (Abbott/ Optimedica, North Chicago, Illinois, USA); Victus (Bausch \& Lomb Rochester, New York, USA); LDV Z8 (Ziemer, Port, Switzerland). The main differences between all laser platforms are the frequency and duration of the energy impulse emission. Victus works with frequency of 80 $\mathrm{kHz}$, an average use power equal to about $7 \mu \mathrm{J}$ and duration of the spot of about 400-550 fs. Catalys works at $120 \mathrm{kHz}$ with an energy average of $4 \mu \mathrm{J}$ and duration of $600 \mathrm{fs}$ spot. The LDV Z8 uses an average frequency of $2 \mathrm{MHz}$, one energy impulse in the range of nano Joules $(50 \mathrm{~nJ}-2500 \mathrm{~nJ})$ and the duration of the unspecified spot; the LenSx uses frequencies equal to $50 \mathrm{kHz}$, a pulse of energy of $15 \mu \mathrm{J}$ and duration of about 600-800 fs. The LensAR laser emission frequency is equal to $80 \mathrm{kHz}$. Catalys allows to run grids in addition to radial cuts, Victus and LenSx have concentric cuts ring and radial, and recently LenSx also to grid pattern. LensAR performs grid and radial cuts with or without concentric ring cuts, while LDV Z8 can only perform radial cuts by default. The FSL can work in various steps of the operations, performing cutting modulated in extension and depth, thus simplifying and making the surgery safer. Generally the FSL are dedicated to only one type of surgery. FSL Visumax (Carl Zeiss, Jena, Germany) and FSL Intralase (Alcon, USA) are used in corneal procedures being the first one, the sole capable to carry out the SMILE technique. The lasers Victus (Bausch and Lomb,) and Femto LDV Z8 Ziemer, Switzerland) are used both in cataract and corneal surgery. FDA approved FSL for cataract surgery in 2010 with indication to make clear corneal incisions, capsulorhexis and lens fragmentation/softening. In the near future a progressive transitioning to the all laser cataract surgery could be expected [1].

\section{FSL clinical applications}

\section{Refractive surgery}

Femtosecond lasers are used in various refractive surgery procedures such as Femtolasik, Femtosecond lenticule extraction (FLEx) and Small-incision lenticule extraction (SMILE)[2]. In the Femtolasik two different lasers are used to perform two surgical stages. During the first one, a corneal flap is created using the FSL, successively the flap is lifted and the next stage consisting in excimer laser ablation is done. In this technique the FSL replaced the microkeratome cut used in the traditional LASIK method to execute the corneal flap (Fig. 3). To create the flap, the laser spot moves inside the cornea at programmed thickness, creating a perfect cut and once the lamella is created, the Femtolaser bundle continues along the flap circumference. This makes the procedure completely blade-free and permits the surgeon to adapt the procedure to the characteristics of each patient, with a better flap thickness predictability. ReLEx (Refractive Lenticule Extraction) is performed entirely with a FSL system. The femtosecond laser cuts a small lenticule of

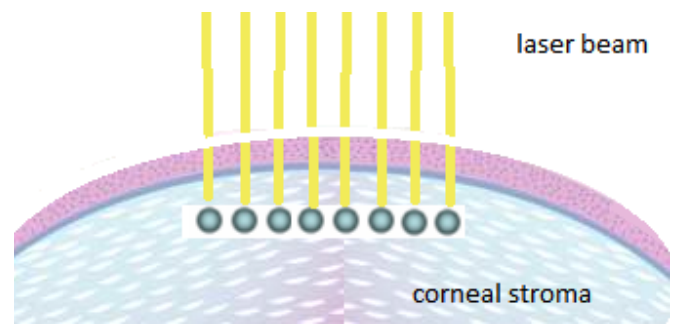

Fig. 1. Each pulse forms a microscopic bubble. The cut is achieved by placing thousand of pulses next to each other.

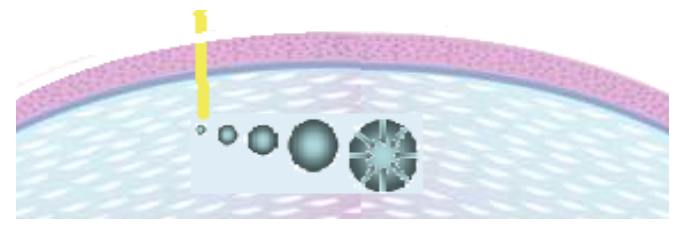

Fig. 2. Expansion of plasma creates cavitation bubbles separating the tissue plane.

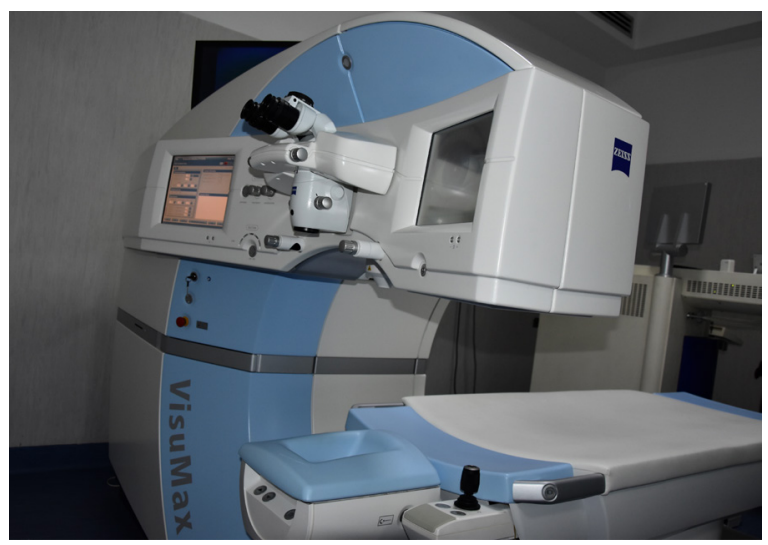

Fig.3. Femtolaser platform.

tissue within the center of the cornea and creates a flap, similar to the flap created in LASIK. The flap is lifted and the lenticule is removed, than the flap is repositioned. The removal of the lenticule reduces the corneal curvature, thereby reducing refractive error. 


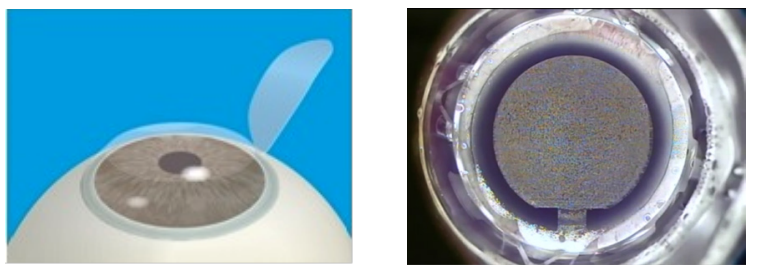

Fig. 3 A, B. Corneal lamellar flap performed by femtosecond laser.

Recently ReLEx was refined to the SMILE procedure that consists in the sculpting of intrastromal lenticule with characteristics adapted to correct the myopic or astigmatic refractive error. FSL performs the anterior and posterior dissection of both faces of the refractive lenticule followed by a small incision in the midperiphery of the cornea, varying from about 2 to $4 \mathrm{~mm}$, allowing the surgeon to extract safely the intrastromal lenticule.

\section{Incision for ICRs}

FSL are used to create intrastromal channels for the intra-corneal ring (ICR) segments implantation used to flatten the central cornea in high myopia or keratoconus. The incision for ICR offers the possibility to create even inclined cutting geometries and tunnel segments between $90^{\circ}$ and $270^{\circ}$.

\section{Corneal surgery}

Corneal surgery assisted with FSL is related to different techniques of transplantation. In fact the laser cuts available the surgeon to create the perfectly matching donor-recipient buttons due to the high precision in corneal incisions. The femtosecond laser-assisted keratoplasty procedure has been developed to produce bladeless, precise, and predefined lamellar cuts with minimal tissue injury [3, 4]. Precise corneal incisions, with customized graft edges and lamellar planes for both donor and recipient corneas can be created by customized FSL trephinations in zig-zag or mushroom or hat conformation [5] (Fig 4).

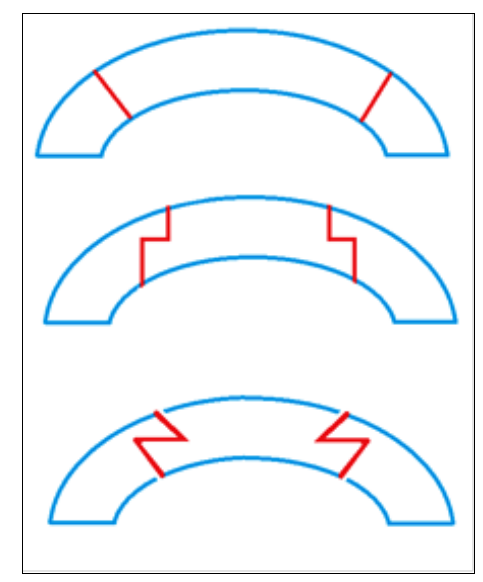

Fig. 4. Possibilities of corneal cut in keratoplasty. The mean central corneal thickness is about $540 \mu \mathrm{m}$ and it ranges from 500 to $600 \mu \mathrm{m}$.

\section{Cataract surgery}

Femtosecond lasers represent an innovative technology and a new frontier in cataract surgery [6, 7]. Many potential benefits are offered by the introduction of the femtosecond laser into the field of cataract surgery. FSL is able to perform three important steps in cataract surgery such as corneal incisions, capsulotomy, and lens fragmentation (Fig. 5). There are currently several laser platforms used for Femto Laser Assisted Cataract Surgery (FLACS). All lasers enter in contact with ocular surface by so called, docking phase, after that, the eye structures are visualized [8]. Once the captured images are confirmed, all platforms are programmed to detect margins of ocular structures before the laser action, for a totally safe cutting. After laser circular capsulotomy, a nucleus fragmentation can be performed through different cutting patterns that are chosen by surgeon accordingly to cataract's hardness. The procedure than is completed manually by the surgeon. LenSx, LensAR and LDV Z8 let to run the whole operation on the same operating bed, moving simply the laser platform with micrometrical resolution.

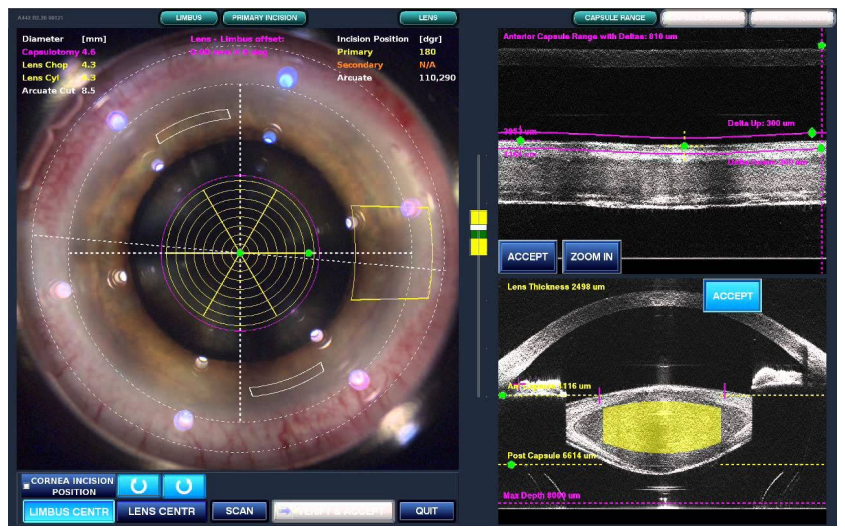

Fig 5. Working plan in FLACS. Right: eye view after docking. Left: Optical Coherence Tomography images of the lens capsule and anterior segment section with cornea and lens (yellow). (Courtesy of dr. F.D’Arrigo)

\section{Discussion}

Ophthalmology was the first medical specialty to utilize laser energy in patient treatment, and still more laser operations than any other specialty are done. The use of femtosecond laser in ocular surgery represents the most advanced and recent technology. This technology, in comparison to the traditional one, does not require the use of blades, therefore resulting less traumatic and ensuring a faster healing. Since their introduction and first human treatment in 2008, by Franz Fankhauser and Daniele Aron-Rosa, significant improvement was achieved. The circular opening of the capsule in the cataract surgery, is perfectly centered and round, thus resulting very important in the post-operative phase when enables a better intraocular lens centration and stability. The liquefaction of softer lenses and the fragmentation of harder lenses allow chopping the nucleus without the use of phaco-energy, in fact there 
were observed a reduction of $39 \%$ to $43 \%$ in the energy and a $51 \%$ decrease in time of phacoemulsification [9]. The most important advantage of femtolaser cataract technology consists in the planning and customization of some important surgical steps. Reduced trauma of the cornea lead to a decreased lost of endothelial cells as compared to conventional phacoemulsification [10-12]. Other advantages in FLACS are standardized corneal incisions, centered and round capsulorhexis, and lens nucleus fragmentation even in eyes with hard cataracts [13]. The laser precision is due to the real time OCT software programs, which cover the whole anterior segment until the posterior lens capsule. Quality of vision can be improved with less posterior chamber lens tilt, more centralized position of the lens, less endothelial damage, less macular edema, and less posterior capsule opacification formation [14-16]. The different cut modalities in corneal transplantation offer many advantages with respect to the traditional keratoplasty. The principle advantages of FSL in this field are faster wound healing, an earlier suture removal, a better donor-host matching and reduced astigmatism. Moreover, femtosecond laser assisted Deep Anterior Lamellar Keratoplasty (DALK) results to be easier to perform as compared to the manual procedure [17]. Corneal grafting with FSL cuts proved to be more predictable and safe technique with better clinical outcomes, due to the laser's precision and repeatable, well controlled, sizing of the donor and recipient corneal buttons [18]. This technique can offer a better centration, a thinner graft/host complex and an earlier corneal healing. As to refractive surgery different advantages of the femtosecond lasers used were reported. In Femtolasik a reduced incidence of flap complications like buttonholes, free caps, irregular cuts are observed [19]. A perfect control of the flap diameter and thickness, side cut angle, hinge position and length, better thickness predictability and increased precision of the flap creation, improved significantly the flap safety and decreased incidence of epithelial ingrowth [20-21]. In fact, several flap-related complications occurring when the conventional microkeratome were used actually are overcome by the femtosecond laser employment. Femtosecond laser creates corneal flaps that have better safety and thickness predictability, their adherence is stronger with respect to the classical manual cut and less influenced by trauma, minimizing the risk of flap displacement and less rate of epithelial ingrowth. SMILE offers additional advantages as no corneal flap is created, and this procedure presents less risk for post-surgical dry eye related to corneal nerves cut and lower incidence of ectasia with respect to LASIK [22-25].

\section{Conclusion}

Wide diffusion of femtosecond lasers is to be expected in ophthalmology in the next future. In fact this technology, working like a blade managed by sophisticated computer software allows the technique to be adapted to each individual case with extreme safety, precision, predictability and repeatability. Moreover FSL work with reduced trauma to the tissues, which results in less inflammation and an earlier recovery. Thanks to the significant impetus of technology, the surgery is moving toward a standardization of surgical procedures and customized approach to each patient.

\section{References}

1. GK. Marino, MR. Santhiago, SE. Wilson. Asia Pac J Ophthalmol. (Phila). 6,5 (2017) G. Sutton, SJ. Bali, C. Hodge. Curr Opin Ophthalmol. 24, 1 (2013)

2. GK. Marino, MR. Santhiago, SE. Wilson. Asia Pac J Ophthalmol. (Phila). 6,5 (2017)

3. M. Taneja, VM. Rathi, B. Bagga. Optom Vis Sci. 92, 2 (2015); 1.

4. A.Aristeidou, E.Taniguchi, M. Tsatsos, R. Muller, C. McAlinden, R. Pineda 4, E. Paschalis. Eye Vis (Lond). 14, 2 (2015)

5. C. Chan, R. Ritenour, N. Kumar., W Sansanayudh., D. Rootman. Cornea. 29, 3 (2010).

6. G. Sutton, SJ. Bali, C. Hodge. Curr Opin Ophthalmol. 24, 1 (2013)

7. ZZ. Nagy. J Refract Surg. 28, 12 (2012).

8. MM. Gavris, R. Belicioiu, I. Olteanu, I. Horge, J. Rom. Ophthalmol. 59,1 (2015)

9. ZZ. Nagy, JP. Szaflik. Klin Oczna.;114, 4 (2012)

10. S. Ganesh, S. Brar. Int J Ophthalmol. 18, 8 (2015)

11. J. Kacerovská, M. Kacerovský, R. Kadlec. Cesk Slov Oftalmol. 695 (2013)

12. AJ. Kanellopoulos, G. Asimellis. Eye Vis (Lond). 29, 3 (2016)

13. M. Pahlitzsch, N. Torun, ML. Pahlitzsch, MKJ. Klamann, J. Gonnermann, E. Bertelmann, T. Pahlitzsch. Semin Ophthalmol. 32, 4. (2017)

14. A. Agarwal, S. Jacob. Curr Opin Ophthalmol. 281 (2017).

15. H. Abouzeid. J Cataract Refract Surg. 428 (2016)

16. Z. Zoltan Nagy. Clin Ophthalmol. 8: 1157-1167. (2014)

17. S. Mariacher, M. Ebner, AM. Seuthe, K. Januschowski, C. Ivanescu, N. Opitz, P. Szurman, K. Boden. J Cataract Refract Surg. 428 (2016)

18. Blériot A, Martin E, Lebranchu P, Zimmerman K, Libeau L, Weber M, Vabres B, Orignac I. J Fr Ophtalmol. 40, 7 (2017)

19. J. Alio, A. Vega-Estrada, F. Soria, A. Abdou. Jaypee Brothers Medical Publishers. 75, 76 (2013)

20. T. Kohnen, OK. Klaproth, V. Derhartunian, D. Kook. Ophthalmologe. 107, 5 (2010)

21. ZS. Bashir, MH. Ali, A. Anwar, MH. Ayub, NH. Butt. J Pak Med Assoc. 674 (2017)

22. D. Touboul, F. Salin, B. Mortemousque, P. Chabassier, E. Mottay, F. Léger, J. Colin. J Fr Ophtalmol. 285 (2005) 
23. S. Taneri, S. Kießler, A. Rost, B. Dick. Klin Monbl Augenheilkd. 2341 (2017)

24. I. Seven, A. Vahdati, IB. Pedersen, A. Vestergaard, J. Hjortdal, CJ. Roberts, WJ Jr Dupps. J Refract Surg. 33,7 (2017).

25. Burazovitch J, Naguzeswski D, Beuste T, Guillard M. J Fr Ophtalmol. 40, 6 (2017)

26. B. Spiru, S. Kling, F. Hafezi, W. Sekundo. Invest Ophthalmol Vis Sci. 58, 5 (2017) 